\title{
Loop-tail phenotype in heterozygous mice and neural tube defects in homozygous mice result from a nonsense mutation in the Vangl2 gene
}

\author{
B. Chen', H.H. Mao' ${ }^{2}$, L. Chen', F.L. Zhang', K. Li ${ }^{1}$ and Z.F. Xue ${ }^{1}$ \\ ${ }^{1}$ Comparative Medicine Center, Yangzhou University, Yangzhou, China \\ ${ }^{2}$ Department of Animal Science and Technology, \\ Jinling Institution of Technology, Nanjing, China \\ Corresponding author: Z.F. Xue \\ E-mail: xuezfyz@yahoo.com.cn
}

Genet. Mol. Res. 12 (3): 3157-3165 (2013)

Received June 14, 2012

Accepted October 13, 2012

Published January 22, 2013

DOI http://dx.doi.org/10.4238/2013.January.22.2

\begin{abstract}
N$-ethyl- $N$-nitrosourea (ENU) is a powerful point mutagen that can generate random mutations. It has been used to generate mouse mutations to produce phenotypic models of human disease. Neural tube defects (NTD) are common birth defects in which the brain and/or spinal cord can be exposed; however, the mechanisms of these defects are poorly understood. Craniorachischisis is one type of NTD that bears a close resemblance to the phenotype of the looptail ( $L p$ ) mouse. Here we describe a C57BL/6J $L p$ mouse generated by ENU-induced mutagenesis. The mutation was mapped to the Vangl2 gene on chromosome 1, near markers D1Mit113 and D1Mit149. Sequence analysis of Vangl2 heterozygotes ( Vangl2 $2^{\mathrm{mlYzcm} /+}$ ) revealed a $\mathrm{C} / \mathrm{T}$ transition mutation that resulted in substitution of a glutamine codon for a stop (nonsense) codon at position 449. The Vangl2 protein is involved in epithelium planar cell polarity. The predicted truncated protein would lack the PDZ-domain binding motif involved in proteinprotein interaction; therefore, Vangl2 ${ }^{\text {mlYzcm }}$ may be a loss-of-function mutant. Morphological and histological examination of homozygous
\end{abstract}


mouse embryos revealed a neural tube closure defect that leads to craniorachischisis. This Vangl2 ${ }^{\text {mlYzcm }}$ mouse represents a valuable model for the study of NTDs in humans.

Key words: $N$-ethyl- $N$-nitrosourea; Loop-tail; Neural tube defect; Vangl2

\section{INTRODUCTION}

Planar cell polarity (PCP) is the process by which cells become polarized in the epithelial plane. This process has been studied in the epithelial tissues of adult Drosophila. Genetic studies have identified a group of so-called 'core' PCP molecules: stbm/vang; frizzled; the intracellular proteins Dishevelled and Prickle; and an atypical cadherin Flamingo, also known as Starry night; diego (Simons and Mlodzik, 2008; Vladar et al., 2009); and the cadherin-related proteins Fat and Dachous (Clark et al., 1995; Matakatsu and Blair, 2004). Vertebrate orthologs of the Drosophila core PCP genes have been identified and recent in vivo studies of mutations that inactivated some of these genes (Vangl2, Celsr l, Scy, Crsh, Dvll, and Dvl2) indicate they regulate convergent extension, a process critical for proper gastrulation and formation of the neural tube (Torban et al., 2004).

$N$-ethyl- $N$-nitrosourea (ENU) is a powerful point mutagen that can generate random mutations in the mouse genome. An ENU-mutagenesis screen for dominant and recessive mutations recovered a large number of mouse mutants with a variety of phenotypes. These mutants provided a rich resource for identifying disease-related genes, deciphering pathogenic mechanisms, and developing new therapies and new drugs. Recently, this procedure established more than 20 lines of mutant mice by screening the ENU-induced mutants in a C57BL/6J background (Wu et al., 2003, 2009, 2010a,b,c; Chen et al., 2011). Interestingly, one of these lines displayed a phenotype similar to that of the Vangl2 (Lpp1) mutant alleles.

In this paper, we report the molecular and phenotypic characterization of the loop-tail $(L p)$ mutant mice. We indentified a C/T transition mutation at position 1345 of Vangl2, which converted a glutamine codon to a stop (nonsense) codon at position 449 in the protein. The mutant mouse was named Vangl2 ${ }^{\mathrm{ml} I z c m}$ (Vangl2; mutation 1, Yangzhou University Comparative Medicine Center). The preliminary phenotypic analysis of the Vangl2 ${ }^{m I Y z c m}$ mouse is described.

\section{MATERIAL AND METHODS}

\section{Ethics statement}

$\mathrm{C} 57 \mathrm{BL} / 6 \mathrm{~J}(\mathrm{~B} 6)$ and $\mathrm{C} 3 \mathrm{H} / \mathrm{HeJ}(\mathrm{C} 3 \mathrm{H})$ mice were obtained from the Shanghai Laboratory Animal Center (Shanghai, China). This study was conducted in strict accordance with the Guide for the Care and Use of Laboratory Animals of the National Research Council. The Animal Care and Use Committee of Yangzhou University approved all animal experiments and procedures (approval ID: SYXK (Su) 2007-0005).

\section{ENU mutagenesis}

Vangl2 $2^{m \text { Yzcm }}$ was isolated as an $L p$ phenotype inherited in a dominant fashion. Wild- 
type B6 males were treated with a $3 \times 100 \mathrm{mg} / \mathrm{kg}$ dose of ENU, allowed to recover fertility, and then mated to B6 females.

\section{Alcian blue and alizarin red staining}

Newborn mice were killed, eviscerated, placed in a $70^{\circ} \mathrm{C}$ water bath for $30 \mathrm{~s}$, and skinned, then fixed in $100 \%$ ethanol for 3 days, followed by staining with alcian blue (15 mg in $80 \mathrm{~mL}$ ethanol $/ 20 \mathrm{~mL}$ glacial acetic acid) for $8-12 \mathrm{~h}$. Skeletons were rinsed in $100 \%$ ethanol overnight and cleared in $2 \% \mathrm{KOH}$ for $6-8 \mathrm{~h}$. Staining for bone was carried out using alizarin red $(50 \mathrm{mg} / \mathrm{L}$ in $2 \% \mathrm{KOH}$ ) for $3-5 \mathrm{~h}$. Skeletons were then cleared in $2 \% \mathrm{KOH}$ and stored in $20 \%$ glycerol.

\section{Linkage analysis}

Vangl2 ${ }^{m I Y z c m}$ heterozygotes of the $\mathrm{B} 6$ background were mated to $\mathrm{C} 3 \mathrm{H}$ mice to generate F1 mice. Next, F1 mice with loop-tail were backcrossed to B6 mice to generate N2 mice. DNA samples of N2 mutant mice were prepared from tail samples by proteinase $\mathrm{K}$ digestion, phenol-chloroform extraction, and ethanol precipitation. DNA samples were screened by polymerase chain reaction (PCR) for microsatellite markers; PCR products were separated on $4 \%$ agarose gels by electrophoresis and analysed.

\section{Sequence analysis of the Vangl2 gene}

The exons and immediate flanking sequences of Vangl2 were amplified from Vangl2 ${ }^{m \text { I Yzcm }}$ heterozygotes and B6 genomic DNA. Primer sequences for Vangl2 are available on request. PCR products were purified and sequences read using the Big Dye Terminator v3.1 kit on an ABI-PRISM 3730 instrument.

\section{Reverse transcription (RT)-PCR}

Total RNA was isolated from the heads of E12.5 embryos using the TRIzol reagent (Invitrogen, Carlsbad, CA, USA). cDNA was synthesized using a RevertAid First-Strand cDNA Synthesis Kit (Fermentas) with oligo(dT)18 primers. RT-PCR for Vangl2 was performed with primers: forward: 5'-TACTACGAGGAAGCCGAGCATGA-3' and reverse: 5'-GCAGCCGCATGACGAACTTATGT-3'. PCR conditions consisted of one cycle of denaturation for $5 \mathrm{~min}$ at $94^{\circ} \mathrm{C}$, followed by 30 cycles of $30 \mathrm{~s}$ at $94^{\circ} \mathrm{C}, 30 \mathrm{~s}$ at $61^{\circ} \mathrm{C}$, and $30 \mathrm{~s}$ at $72^{\circ} \mathrm{C}$, ending with one cycle of elongation for $5 \mathrm{~min}$ at $72^{\circ} \mathrm{C}$. PCR products were purified and sequenced.

\section{Genotyping}

The point mutation introduced an FspBI (BfaI) restriction site in Vangl2 ${ }^{\text {mIYzcm }}$. A 452-bp fragment that encompassed the point mutation was amplified from genomic DNA using forward primer: 5'-AAACACCCTAGCTATCTTAGAAAG-3' and reverse primer: 5'-GGAAGTAGGACTGGCAGAAATGTG-3'. Digestion of the PCR product with FspBI was predicted to yield DNA fragments of the following sizes: +/+ mice, $452 \mathrm{bp} ;+/$ - heterozygotes, 
452, 133, and 319 bp; and -/- homozygous mice, 133 and 319 bp. Analysis after 2\% gel electrophoresis revealed DNA bands of the expected sizes.

\section{Histology}

Heterozygous Vangl2 ${ }^{m l Y z c m}$ mice were intercrossed and embryos at E12.5 and E18.5 were recovered, genotyped, and examined for the presence of neural tube defects (NTDs). Embryos at E12.5 were fixed in 4\% paraformaldehyde in phosphate-buffered saline, dehydrated, wax-embedded and sectioned at $10 \mu \mathrm{m}$, and then stained with Harris hematoxylin and eosin-Y stain.

\section{RESULTS}

\section{Phenotype of Vangl2 ${ }^{m 1 Y z c m}$ heterozygous mice}

The founder male mouse with an $L p$ appearance (Figure 1) was the progeny of an ENU-treated B6 male mouse. The mutant mouse was mated with B6 mice: 32/103 of the progeny produced had the $L p$ phenotype. The $\mathrm{B} 6$ heterozygotes were mated to $\mathrm{C} 3 \mathrm{H}$ mice to generate F1 mice: $1 / 32$ of the progeny had the $L p$ phenotype, indicating the presence of a background effect. Alcian blue and alizarin red staining showed that the loop-tail phenotype was caused by a contortion of the tail, and the skeletons of the heterozygotes were normal (Figure 2).

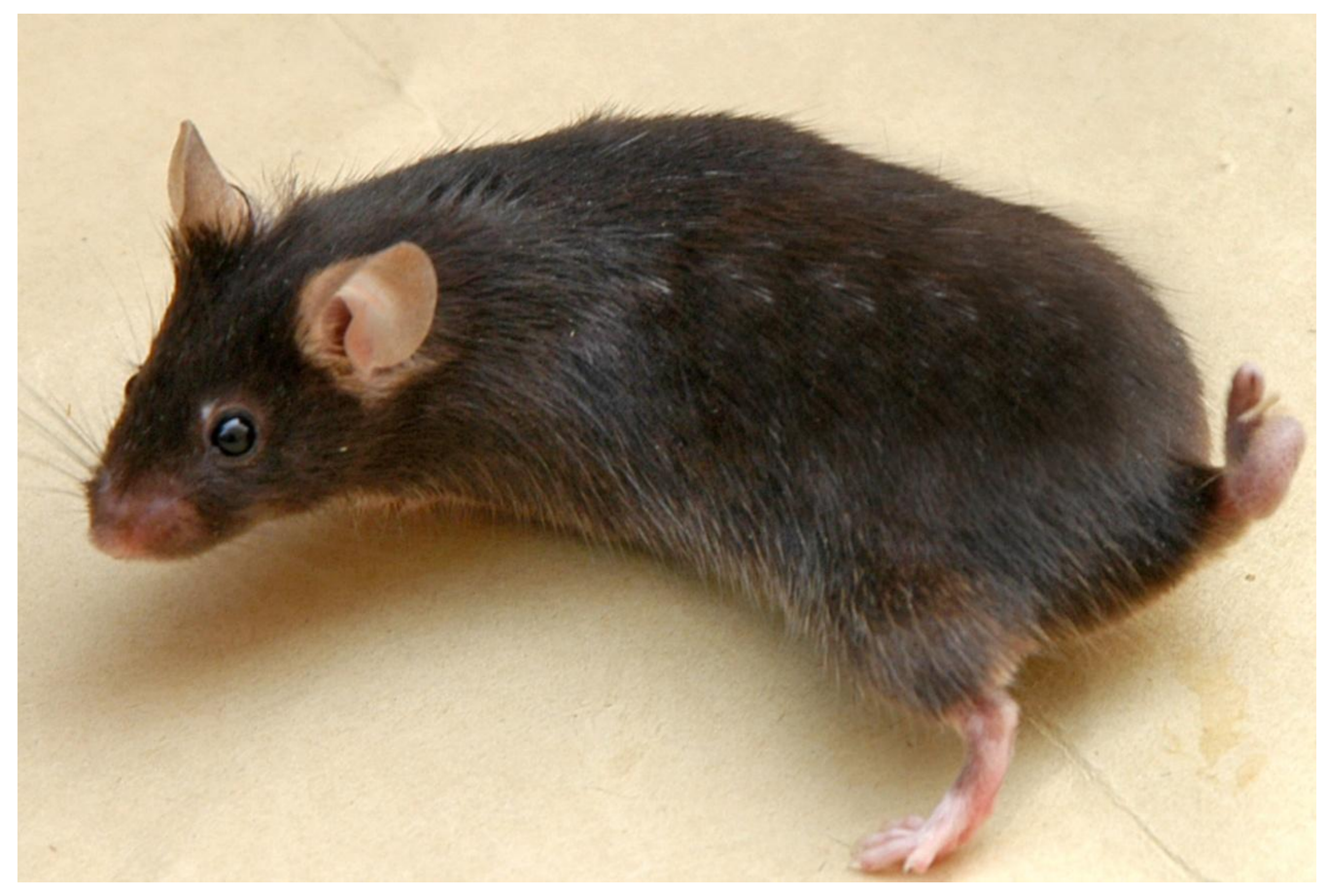

Figure 1. Loop-tail appearance of Vangl2 ${ }^{m I Y z c m}$ heterozygous mouse. 


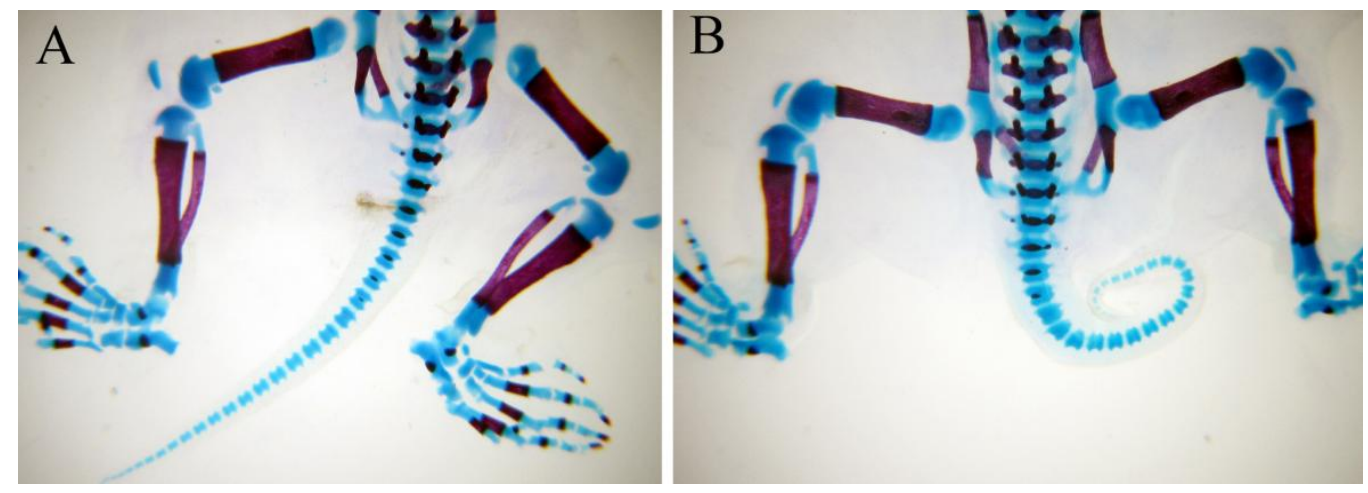

Figure 2. Alcian blue and alizarin red staining. A. Wild-type mouse. B. Vangl2 ${ }^{m l Y z c m}$ heterozygous mouse.

\section{Genetic mapping}

We tested genomic DNA from 42 N2 samples by mapping microsatellite markers. Previous descriptions of the chromosomal location of the $L p$ mutant genes suggested that some microsatellite markers near these genes were used. The mutation was mapped to chromosome 1 and had no exchange with markers D1Mit113 and D1Mit149, which were located at 79.54 and $80.13 \mathrm{cM}$, respectively. An $L p$-associated gene, Vangl2, $79.54 \mathrm{cM}$ from the centromere on chromosome 1, was chosen to be a particularly good candidate for mutation.

\section{$L p$ phenotype is caused by a nonsense mutation in the Vangl2 gene}

On examination of the Vangl2 gene, we found a single nucleotide change, a C/T substitution, in exon 8 at nucleotide position 1345. This substitution converted tyrosine to glutamine to a nonsense mutation at position 449 in the protein (Figure 3A, B, and C). The point mutation introduced an FspBI (BfaI) restriction site in $\mathrm{Vangl}^{\mathrm{ml} \text { Yzcm }}$.

Sequencing of Vangl2 RT-PCR products from Vangl2 ${ }^{\mathrm{ml} Y z c m}$ heterozygous mutant mice showed that the mutant allele is transcribed, correctly spliced, and not subject to nonsense-mediated decay (Figure 3D). Translation of the mutant allele would produce a truncated protein.

\section{Phenotype of the Vangl2 ${ }^{m 1 Y_{z} c m}$ homozygous mice}

Embryos from Vangl2 ${ }^{m I Y z c m}$ brother-sister matings were isolated at E12.5 and E18.5, genotyped (Figure 4), and studied to characterize the Vangl2 ${ }^{m I Y c m}$ phenotype. Several homozygous E18.5 embryos (4/16) were isolated and had an apparent NTD. Morphological examination of the homozygous mutant embryos revealed a malformed neural tube that was open from midbrain to tail and was clearly distinguishable from the closed neural tube of wild-type littermates; embryos also had failure of eyelid closure. The NTD in these embryos was also associated with smaller overall size, suggesting overall growth retardation (Figure 5). Histological examination of transverse sections through Vangl2 ${ }^{\mathrm{mlYccm}}$ homozygous mutant embryos at E12.5 illustrated defects that included an enlarged floor plate and exposed neuroepithelium (Figure 6). 
A

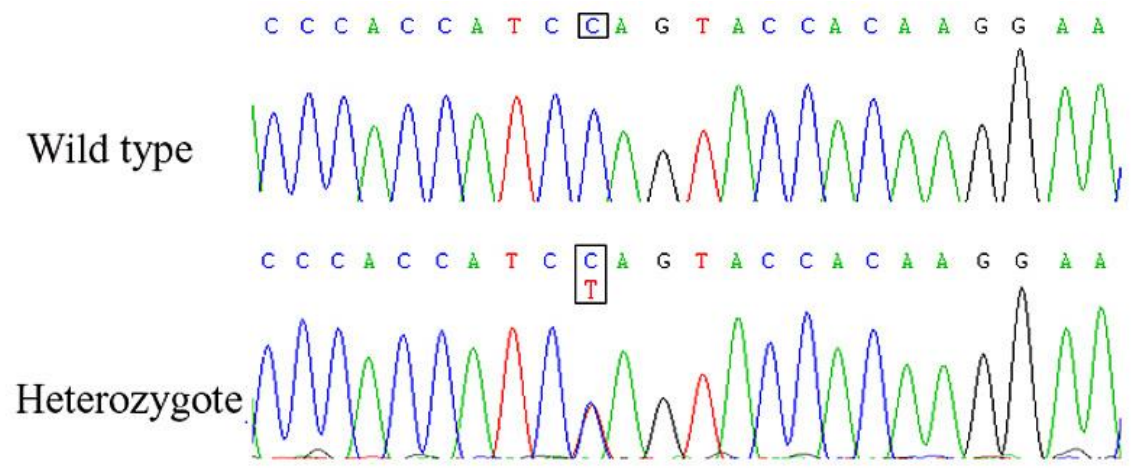

$\mathrm{B}$
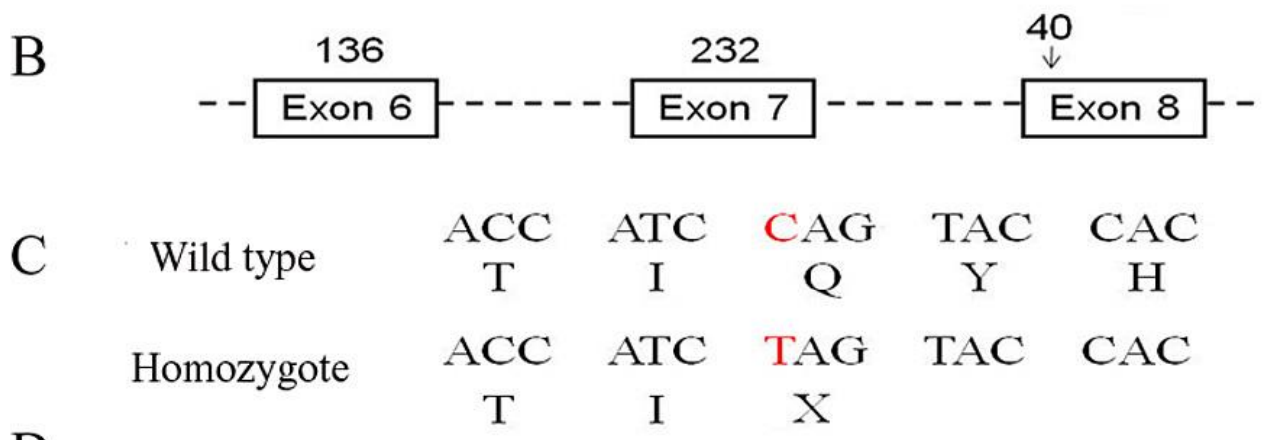

$\mathrm{D}$

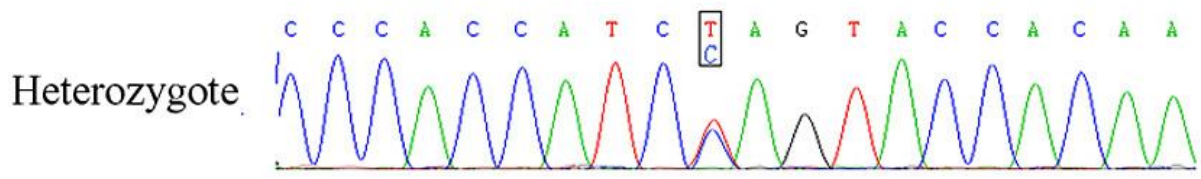

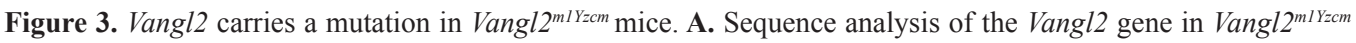
heterozygous mice by ABI-PRISM 3730. B. Partial exon and intron structure of the mouse Vangl2 gene. The arrow shows the position of the Vangl2 $2^{m l Y z c m}$ substitution. C. Translation of wild-type and homozygous Vangl2 $2^{m l Y z c m}$ alleles flanking the mutation site. The nucleotide mutated in the Vangl2 ${ }^{m I Y z c m}$ allele is highlighted in red. D. Sequence analysis of reverse-transcription polymerase chain reaction products of Vangl2 in Vangl2 ${ }^{\text {mlYzcm }}$ heterozygous mice.

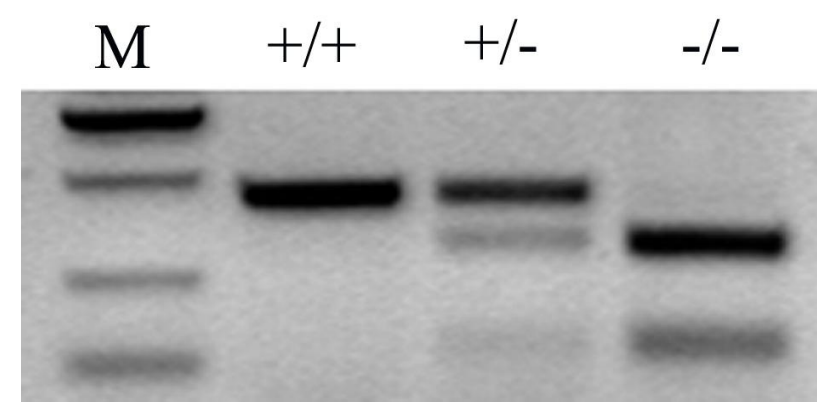

Figure 4. Genotyping of embryos from Vangl $2^{m l Y z c m}$ brother-sister matings. Lane $M=$ molecular weight markers; lane $+/+=$ wild-type mouse; lane $+/-=$ heterozygous mouse; lane $-/-=$ homozygous mouse. 

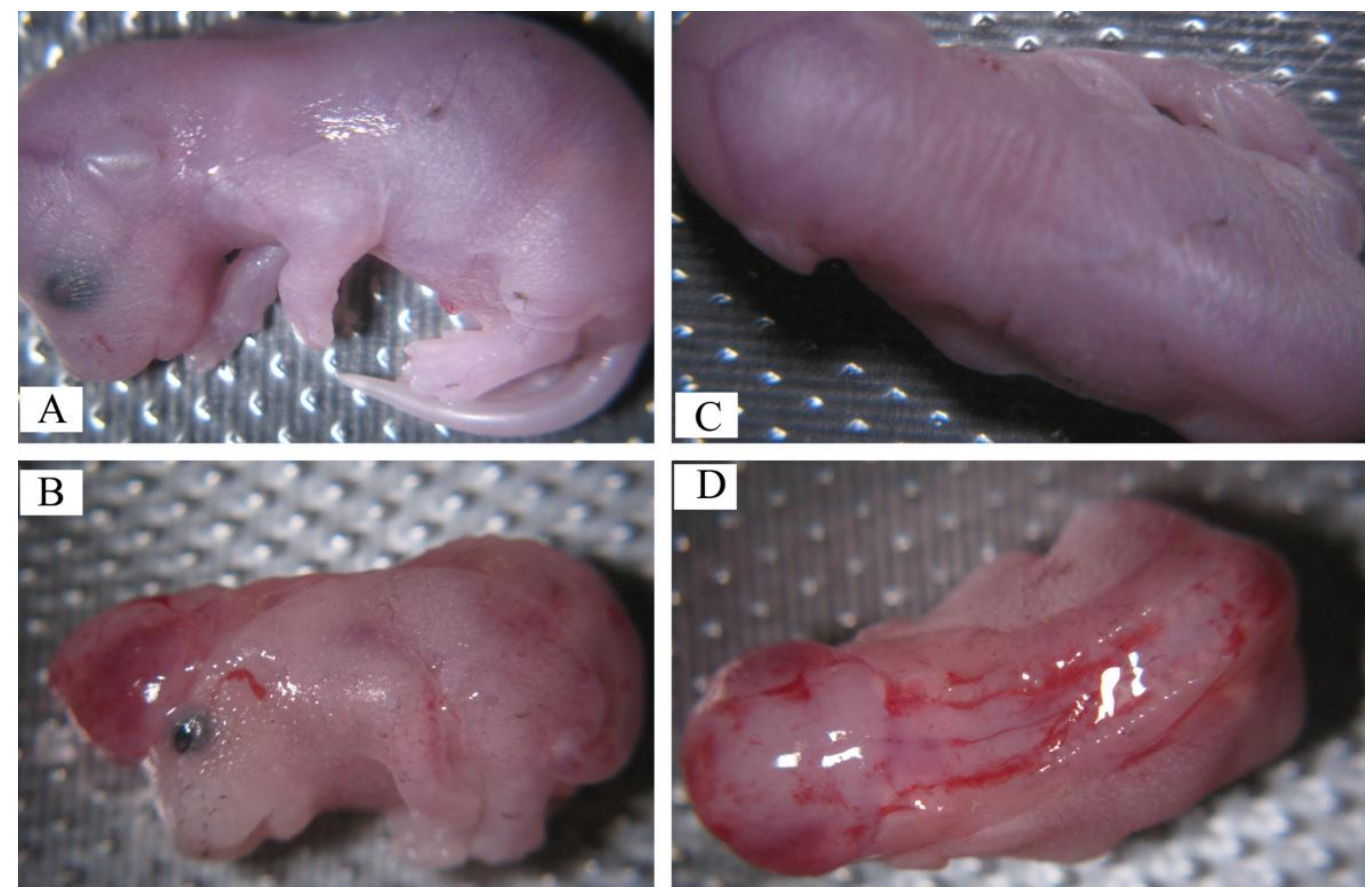

Figure 5. Morphological appearance of $\operatorname{Vangl2^{mlYzcm}}$ homozygous embryos lateral (A, B) and dorsal (C, D) views of wild-type $(\mathbf{A}, \mathbf{C})$ and $\operatorname{Vangl}^{m 1 Y z c m} / \operatorname{Vangl} 2^{m 1 Y z c m}(\mathbf{B}, \mathbf{D})$ E18.5 embryos.
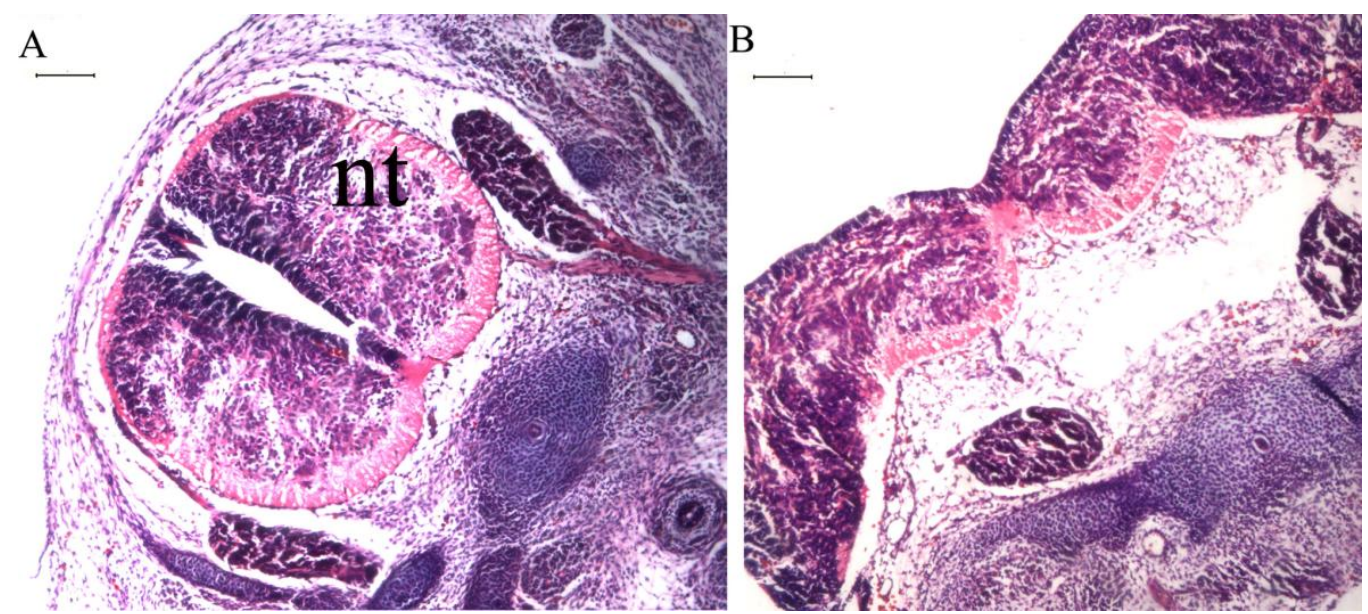

Figure 6. Histological examination of transverse sections through wild-type (A) and homozygous mutant Vangl2 $2^{m l Y_{z c m}} / \operatorname{Vangl2^{mlYzcm}}(\mathbf{B})$ embryos at E12.5. Sections were stained with hematoxylin and eosin. $\mathrm{nt}=$ neural tube. Scale bars $=100 \mu \mathrm{m}$. 


\section{DISCUSSION}

Closure of the neural tube is essential for normal development of the brain and spinal cord. Failure of correct neural tube formation is one of the most common structural malformations of the central nervous system in humans and affects 1-2 infants per 1000 births (Copp et al., 1990; Harris and Juriloff, 1999; Kibar et al., 2001).

ENU-induced mutagenesis represents a powerful tool for the study of gene function and generation of human disease models. Three independent mutant alleles have been described for $L p$ mice: the naturally occurring $L p$ (Strong and Hollander, 1949) and the chemically induced $L p^{m I J u s}$ and $L p^{m 2 J u s}\left(s k a^{17}\right)$. Three mutations, S464N, D255E, and R259L, were identified in $L p, L p^{m I J u s}$ and $L p^{m 2 J u s}$, respectively (Murdoch et al., 2001; Guyot et al., 2011).

We identified a novel ENU-induced $L p$ mutant mouse. The underlying genetic defect responsible for the $L p$ phenotype of Vangl2 ${ }^{\text {mIYzcm }}$ mice is a nonsense mutation in exon 8 of the Vangl2 gene, which, if translated, produces a truncated, 448-amino acid protein product. Vangl2 (Lpp 1) encodes a full-length protein of 521 amino acids, with 4 transmembrane domains that are related to the Drosophila protein Van Gogh (Vang) and a large intracellular domain with a PDZ-domain binding motif [post synaptic density protein (PSD95), Drosophila disc large tumor suppressor (Dlg1), and zonula occludens-1 protein (zo-1)] at its carboxy terminus, which mediates protein-protein interaction (Murdoch et al., 2001; Torban et al., 2004). The predicted truncated protein product, therefore, lacks the PDZ-domain binding motif. We predict, therefore, that $\mathrm{Vangl}^{\mathrm{mlYzcm}}$ is a loss-of-function mutant.

Vangl2 ${ }^{m I Y z c m}$ heterozygous mice are characterized by an $L p$ appearance, while homozygous embryos show a severe form of NTD called craniorachischisis, in which the neural tube remains open throughout the hindbrain and spine. Thus, the allelic series of mutations in the Vangl2 gene represent an extremely valuable genetic resource for understanding the function of this gene in PCP signaling. The Vangl2 ${ }^{m l Y z c m}$ mouse represents a valuable model for the study of NTDs in humans.

\section{ACKNOWLEDGMENTS}

This paper was edited by a native English professional with a science background at Elixigen Corporation. Research supported by grants from the National Natural Science Foundation of China (\#31000987) and the Priority Academic Program Development of Jiangsu Higher Education Institutions (PAPD).

\section{REFERENCES}

Chen B, Li K, Zhang F, Zhai G, et al. (2011). An ENU-induced mutation of Nrgl causes dilated pupils and a reduction in muscarinic receptors in the sphincter pupillae. PLoS One 6: e25176.

Clark HF, Brentrup D, Schneitz K, Bieber A, et al. (1995). Dachsous encodes a member of the cadherin superfamily that controls imaginal disc morphogenesis in Drosophila. Genes Dev. 9: 1530-1542.

Copp AJ, Brook FA, Estibeiro JP, Shum AS, et al. (1990). The embryonic development of mammalian neural tube defects. Prog. Neurobiol. 35: 363-403.

Guyot MC, Bosoi CM, Kharfallah F, Reynolds A, et al. (2011). A novel hypomorphic Looptail allele at the planar cell polarity Vangl2 gene. Dev. Dyn. 240: 839-849.

Harris MJ and Juriloff DM (1999). Mini-review: toward understanding mechanisms of genetic neural tube defects in mice. Teratology 60: 292-305. 
Kibar Z, Underhill DA, Canonne-Hergaux F, Gauthier S, et al. (2001). Identification of a new chemically induced allele ( $\left.L p^{m l J u s}\right)$ at the loop-tail locus: morphology, histology, and genetic mapping. Genomics 72: 331-337.

Matakatsu H and Blair SS (2004). Interactions between Fat and Dachsous and the regulation of planar cell polarity in the Drosophila wing. Development 131: 3785-3794.

Murdoch JN, Doudney K, Paternotte C, Copp AJ, et al. (2001). Severe neural tube defects in the loop-tail mouse result from mutation of Lpp1, a novel gene involved in floor plate specification. Hum. Mol. Genet. 10: 2593-2601.

Simons M and Mlodzik M (2008). Planar cell polarity signaling: from fly development to human disease. Annu. Rev. Genet. 42: 517-540.

Strong LC and Hollander WF (1949). Hereditary loop-tail in the house mouse accompanied by imperforate vagina and with lethal craniorachischisis when homozygous. J. Hered. 40: 329-334.

Torban E, Kor C and Gros P (2004). Van Gogh-like2 (Strabismus) and its role in planar cell polarity and convergent extension in vertebrates. Trends Genet. 20: 570-577.

Vladar EK, Antic D and Axelrod JD (2009). Planar cell polarity signaling: the developing cell's compass. Cold Spring Harb. Perspect. Biol. 1: a002964.

Wu BJ, Mao HH, Shao YX, Xue ZF, et al. (2003). Four kinds of ENU-induced white spot mice and chromosome locations of the mutant genes. Chinese Sci. Bull. 48: 2658-2664.

Wu BJ, Yin LJ, Yin XS, Yang WW, et al. (2009). Homozygous lethality and heterozygous spotting due to a novel missense mutation in the mouse Kit gene. Curr. Zool. 55: 430-434.

Wu BJ, Yin LJ, Yin HP, Ying XS, et al. (2010a). A mutation in the Kit gene leads to novel gonadal phenotypes in both heterozygous and homozygous mice. Hereditas 147: 62-69.

Wu BJ, Shao Y, Chen B, Liu C, et al. (2010b). Identification of a novel mouse brachyury $(T)$ allele causing a short tail mutation in mice. Cell Biochem. Biophys. 58: 129-135.

Wu BJ, Zeng YM, Mao HH, Yin LJ, et al. (2010c). Mapping of genetic modifiers of Plcdl in scant hair mice (snthrlBao). Chinese Sci. Bull. 55: 4026-4031. 